21st Particles and Nuclei International Conference (PANIC 2017)

International Journal of Modern Physics: Conference Series

Vol. 46 (2018) 1860073 (7 pages)

(C) The Author(s)

DOI: $10.1142 / \mathrm{S} 201019451860073 \mathrm{X}$

\title{
Large Scale Beam-Tests of the Silicon and Scintillator-SiPM Modules for the CMS High Granularity Calorimeter at the HL-LHC
}

\author{
Shilpi Jain (on behalf of the CMS Collaboration) \\ Department of Physics, National Central University, No. 300, \\ Zhongda Road, Zhongli District, Taoyuan City, 320 Taiwan \\ shilpi.jain@cern.ch
}

Published 3 May 2018

\begin{abstract}
The High Granularity Calorimeter (HGCAL) will replace the existing CMS endcap calorimeters during the High Luminosity run of the LHC (HL-LHC) era. The electromagnetic part, as well as the first layers of the hadronic part, foresees around 600 square metres of silicon sensors as the active material. The remainder of the HGCAL, in the lower radiation environment, will use plastic scintillators with on-tile silicon photomultiplier (SiPM) readout. Prototype hexagonal silicon modules, featuring a new ASIC (Skiroc2-CMS), together with a modified version of the scintillator-SiPM CALICE AHCAL, have been tested in beams at CERN. This setup represents a full slice through HGCAL. Results from MIP calibration, energy resolution, electromagnetic and hadronic shower-shapes are presented using electrons, pions and muons.
\end{abstract}

Keywords: HL-LHC; HGCAL; CMS; Silicon; Skiroc2-CMS; AHCAL.

\section{Introduction}

The endcap CMS electromagnetic and hadronic calorimeter would face neutron fluences of $10^{16}$ neutrons $/ \mathrm{cm}^{2}$ for an integrated luminosity of $3000 \mathrm{fb}^{-1}$ in the region of $|\eta| \sim 3$. Such a high radiation environment will cause irrecoverable radiation damage to the current endcap calorimeter. Also, the average number of expected primary vertices will rise up to $\sim 200$ in a single bunch crossing. Such a high pileup causes problems in the object reconstruction and identification. Upon seriously considering the issue, CMS has proposed the replacement of the current endcap calorimeters with a new high-granularity $(\mathrm{HGCAL})^{1}$ sampling calorimeter in the range $1.5<|\eta|<3.0$ to address these challenges. The proposed design includes a silicon/lead electromagnetic section followed by two hadronic sections using stainless steel as the absorber material. In the front hadronic section, silicon is used as the

This is an Open Access article published by World Scientific Publishing Company. It is distributed under the terms of the Creative Commons Attribution 4.0 (CC-BY) License. Further distribution of this work is permitted, provided the original work is properly cited. 
active material, whereas, the back sections use both silicon and plastic scintillator as active material. This is also discussed in detail in this reference. ${ }^{2}$ In the beam tests performed during 2016, a prototype of the electromagnetic section (CE-E) with hexagonal silicon pad sensors was built and tested at both FNAL and CERN to explore the calorimetric performance of the electromagnetic part. In the beam tests at FNAL, a 16-layer $\left(0-15 X_{0}\right)$ system was built and tested with electron energies ranging between 4-32 GeV. At CERN, an 8-layer system (up to $27 X_{0}$ ) was tested with electrons of energies between 20 and $250 \mathrm{GeV}$. The silicon modules in these tests used Silikon Kalorimeter Read-Out Chip (SKIROC) ${ }^{3}$ as the front-end ASIC with a double layered module hexagonal PCB (hexaboard) that contains the front end ASICs and provides connections via wire-bonds through holes to the silicon. In 2017, we tested a prototype of the electromagnetic (CE-E) and the hadronic (CE-H(Si) and $\mathrm{CE}-\mathrm{H}($ Scintillator)) sections. With this configuration, we explored hadronic showers for the first time with a prototype HGCAL system. A system was built with two layers, each layer having one module $\left(\sim 17 X_{0}\right)$ in CE-E, 4 layers having a total of 8 modules $(\sim 4.4 \lambda)$ in $\mathrm{CE}-\mathrm{H}(\mathrm{Si})$ and 12 active layers $(\sim 5 \lambda)$ in $\mathrm{CE}-\mathrm{H}$ (Scintillator). The silicon modules in these tests used SKIROC2-CMS ${ }^{4}$ as the front-end ASIC which is better suited to the specific needs of CMS with a shaping time of $40 \mathrm{~ns}$. We also used a new on-detector single layer hexaboard as opposed to a two layered PCB module in 2016 as it is closer to the design of the proposed HGCAL. Though the original goal of the beam-tests this year was to have 28 layers in CE-E and 12 layers with 7 modules each in $\mathrm{CE}-\mathrm{H}(\mathrm{Si})$, for the July beam-tests, we managed to test 2 modules in CE-E and 8 modules in $\mathrm{CE}-\mathrm{H}(\mathrm{Si})$. The main bottleneck was the hexaboard production and thus the setup had to be reduced compared to the planned goal. This report outlines a brief summary of the results from 2016 and their comparison with simulation obtained using GEANT4. It also discusses the preliminary results from the 2017 test-beam at CERN. FTFP_BERT_EMM was used as the physics list for comparison of test beam results with GEANT4. More detailed results from the 2016 beam-tests can be found in this reference. ${ }^{5}$ At the time of this conference, beam-tests performed in July, 2017 at CERN, were available. These results are also discussed in this report. More beam-tests were performed in the latter part of 2017 and are hence not included in this report.

\section{The HGCAL prototype}

\section{1. $C E-E$ and $C E-H(S i)$}

The sensors used in the beam-tests in CE-E and $\mathrm{CE}-\mathrm{H}(\mathrm{Si})$ are 128-channel hexagonal devices made from $6^{\prime \prime} \mathrm{p}$-in-n silicon wafers. The physical thickness of these sensors is $320 \mu \mathrm{m}$ with a depleted thickness of $200 \mu \mathrm{m}(300 \mu \mathrm{m})$ in the $2016(2017)$ beam-tests. The modules used in the construction of $\mathrm{CE}-\mathrm{E}$ and $\mathrm{CE}-\mathrm{H}(\mathrm{Si})$ are comprised of the following: a $\mathrm{CuW}(25 \%-75 \%)$ base plate, a Kapton sheet, a silicon sensor and a PCB which facilitates wire bonds down to the sensor. In the 2016 

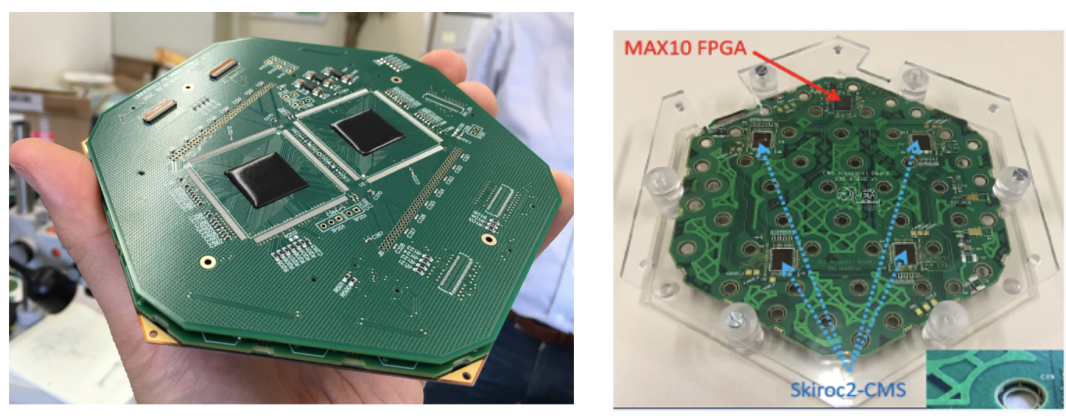

Fig. 1. Complete assembled module for the 2016 beam-tests with double PCB layer readout and two SKIROC ASICs (left) and with a single PCB layer for the 2017 beam-tests with four SKIROC2-CMS ASICs (right).

beam-tests, a second PCB (with two SKIROC ASICs) was plugged into connectors on this first PCB. For the 2017 beam-tests, four SKIROC2-CMS ASICs were mounted on the single layer PCB. Figure 1 shows a fully constructed module used in the 2016 and 2017 beam-tests.

\subsection{CE-H(Scintillator)}

This section of the hadronic calorimeter is a modified version of the CALICE AHCAL (ANalog Hadron Calorimeter). ${ }^{6}$ It is a steel sandwich that uses $3 \times 3 \times 0.3 \mathrm{~cm}^{3}$ scintillating tiles, with the scintillation light directly read out by SiPM detectors from each tile individually. A total of twelve layers of $74 \mathrm{~mm}$ steel absorber plates were interspersed by planes of scintillator tiles. Each active layer has 144 scintillator tiles.

\subsection{Experimental setup}

The modules used in the CE-E and CE-H(Si) were attached to $6 \mathrm{~mm}\left(0.4 X_{0}\right)$ thick copper plates. In the 2016 FNAL (CERN) beam-tests which consisted only of CE$\mathrm{E}$, sixteen modules (eight modules) were interspersed with tungsten absorbers. The total thickness of the setup at FNAL was limited to $15 X_{0}$. Whereas at CERN, two setups were explored: one having the modules placed between about $6 X_{0}$ and $15 X_{0}$, whereas the other having modules covering longitudinal depths between $5 X_{0}$ and $27 X_{0}$. In 2017, beam-tests were performed only at CERN. Figure 2 shows the relative arrangement of $\mathrm{CE}-\mathrm{E}, \mathrm{CE}-\mathrm{H}(\mathrm{Si})$ and $\mathrm{CE}-\mathrm{H}$ (Scintillator) in the CERN test-beam area. In CE-E, the absorber plates (closer to the design of the proposed HGCAL) were made of Fe-Pb-Fe sandwich $(0.3 \mathrm{~mm}$ of $\mathrm{Fe}$ and $4.9 \mathrm{~mm}$ of $\mathrm{Pb})$, whereas in $\mathrm{CE}-\mathrm{H}(\mathrm{Si})$, the absorbers consisted of $40 \mathrm{~mm}$ Fe Plates. Two Si modules were placed in CE-E at $\sim 6 X_{0}, 0.35 \lambda$ and $\sim 17 X_{0}, 0.9 \lambda$. The $\mathrm{CE}-\mathrm{H}(\mathrm{Si})$ composed of a total of eight $\mathrm{Si}$ modules in four layers. The first two layers which were placed at $\sim 25 X_{0}, 1.66 \lambda$ and $\sim 33 X_{0}, 2.4 \lambda$ had a single module each. On the other hand, the last two layers placed at $\sim 41 X_{0}, 3.3 \lambda$ and $\sim 48.5 X_{0}, 4.1 \lambda$ had three modules each. 
S. Jain

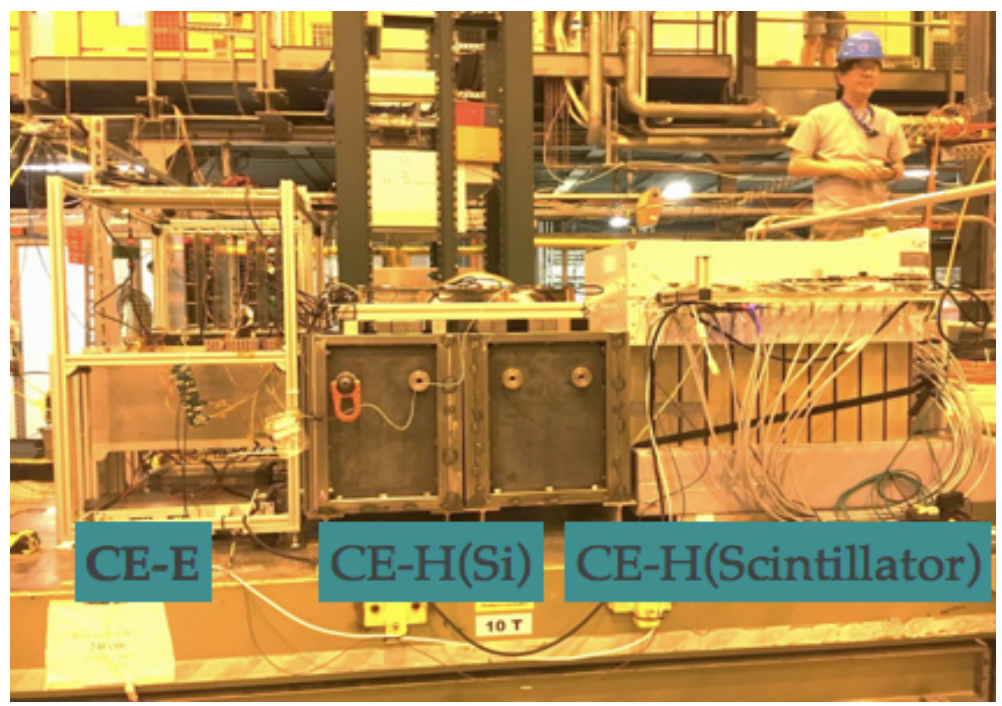

Fig. 2. Relative arrangement of CE-E, CE-H(Si) and CE-H(Scintillator) in the CERN test-beam area during 2017.

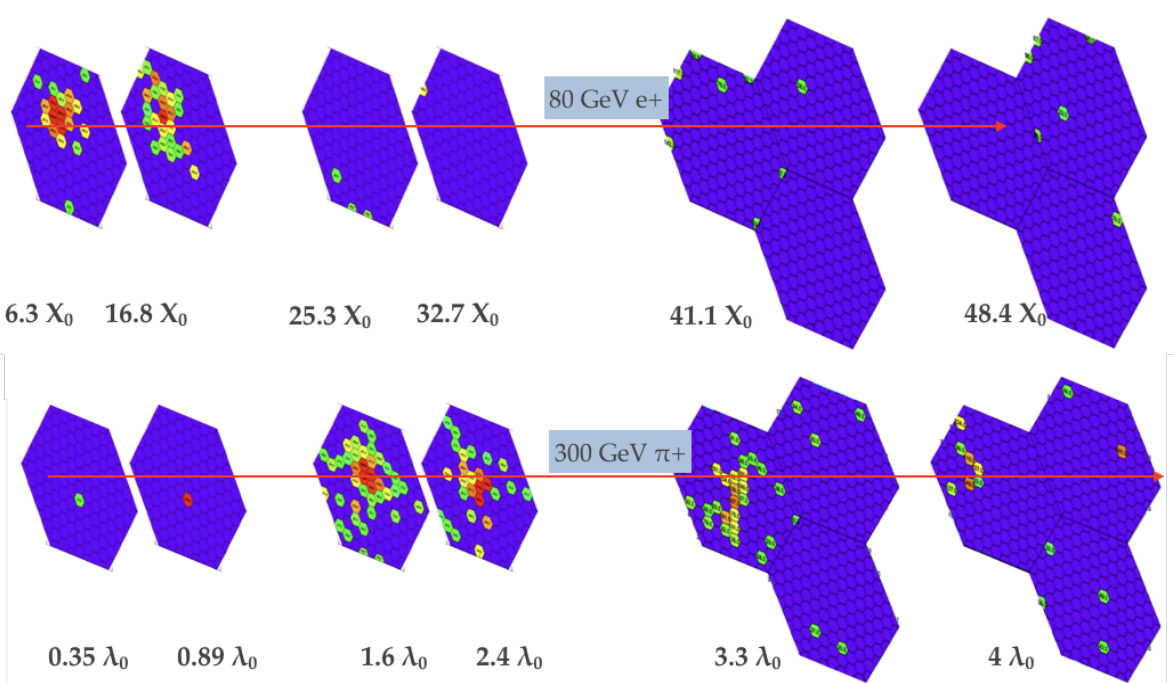

Fig. 3. Shower formation from $80 \mathrm{GeV}$ electrons (top) and $300 \mathrm{GeV}$ pions (bottom) for the 2017 beam-tests.

\section{Results}

Figure 3 shows the shower formation from $80 \mathrm{GeV}$ electrons and $300 \mathrm{GeV}$ pions in the $\mathrm{CE}-\mathrm{E}$ and $\mathrm{CE}-\mathrm{H}(\mathrm{Si})$ layers from the 2017 beam-tests. 


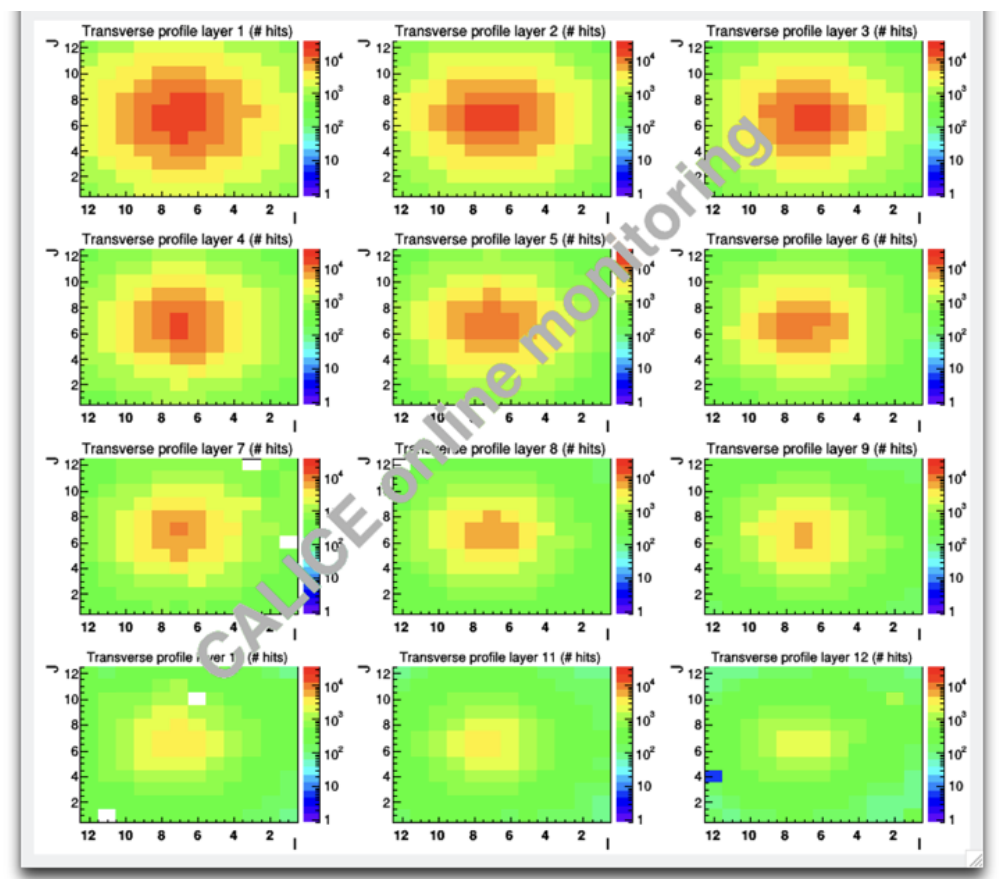

Fig. 4. Number of hits in each layer in CE-H(Scintillator) from a $300 \mathrm{GeV}$ pion run for the 2017 beam-tests. It shows how the shower evolves with the layer in CE-H(Scintillator).

Figure 4 shows the number of hits in each of the twelve layers in the CE$\mathrm{H}$ (Scintillator) layer from a $300 \mathrm{GeV}$ pion run. It is reconstructed from CALICE online monitoring. It shows the evolution of the shower in each layer in CE$\mathrm{H}$ (Scintillator).

To calibrate the detector (i.e., to obtain the $\mathrm{ADC} \rightarrow \mathrm{MIP}$ conversion factor), $125 \mathrm{GeV}$ pions were used which provided "MIPs" (minimum ionising particles) in the modules. Figure 5 shows the MIP distribution in CE-E section in terms of ADC counts for the 2016 beam-tests at CERN. The MIP signal is modelled with a Gaussian convoluted with a sum of Landau distributions to model one MIP and two MIP signals; and the pedestal distribution is modelled with a simple Gaussian function. The same figure also shows the MIP distribution in the $\mathrm{CE}-\mathrm{H}$ (Scintillator) from the 2017 run after selecting single particles with a minimal track-stub selection within hadron showers. This plot is shown after a preliminary calibration.

To understand how well the data is described by simulation, appropriate variables are the relative energy resolution as a function of the beam energy (shown in Figure 6) and the transverse shower spread. To estimate the transverse shower spread, the variables E1/E7 and E7/E19 are examined. E7 (E19) is the sum of energy in the most energetic cell (E1) plus that in the surrounding ring (two rings) 

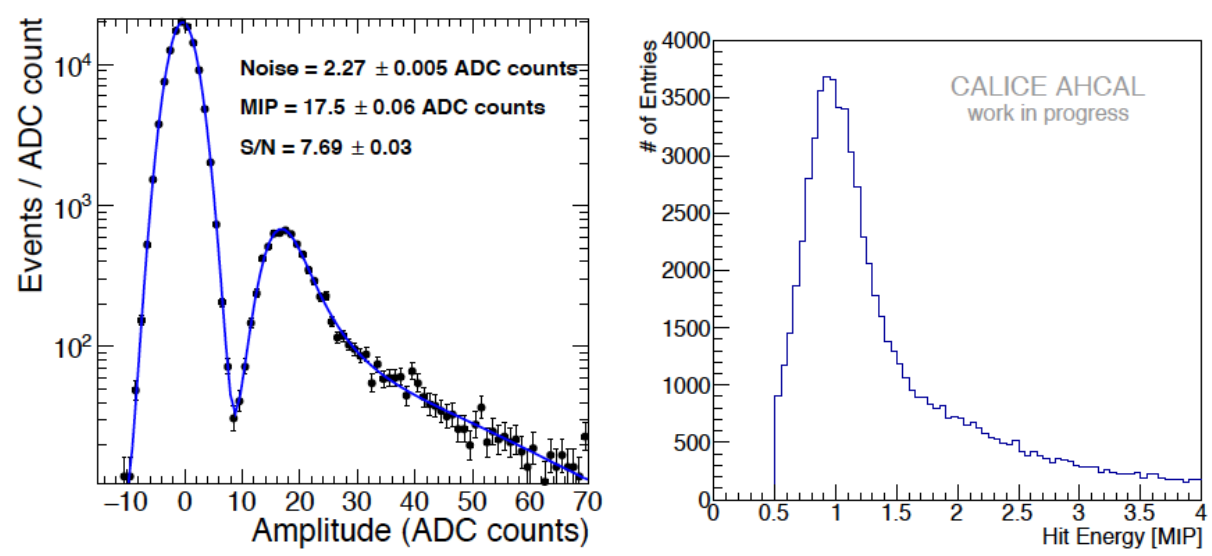

Fig. 5. Left: Energy distribution from MIPs in terms of ADC counts in the CE-E section from 2016 beam tests at CERN. The left peak corresponds to the pedestal distribution and the right peak to the MIP signal. Right: energy spectrum measured in MIPs in CE-H(Scintillator) due to single charged particles in hadron showers after a preliminary calibration.

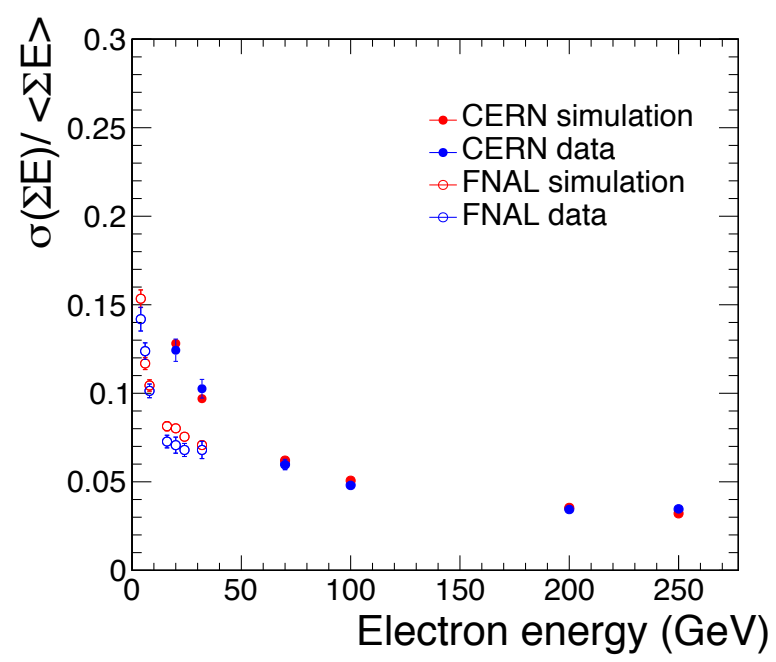

Fig. 6. Relative energy resolution as a function of the beam energy for both data (blue) and simulation (red). Data matches with simulation within $\approx 5 \%$. The limited number of samplings, and limited overall depth for higher energy electrons limit the energy resolution. This is shown from the 2016 beam-test at FNAL and CERN.

of $\operatorname{six}($ eighteen) cells. Figure 7 shows the ratio of E1/E7 in data (2016) and simulation for incident $100 \mathrm{GeV}$ electrons at a depth of about $10 X_{0}$, and the ratio E7/E19 (2017 beam-tests) for incident $200 \mathrm{GeV}$ charged hadrons, at a depth of around $4 \lambda$. A decent agreement can be seen between the data and simulation for both shower types. 

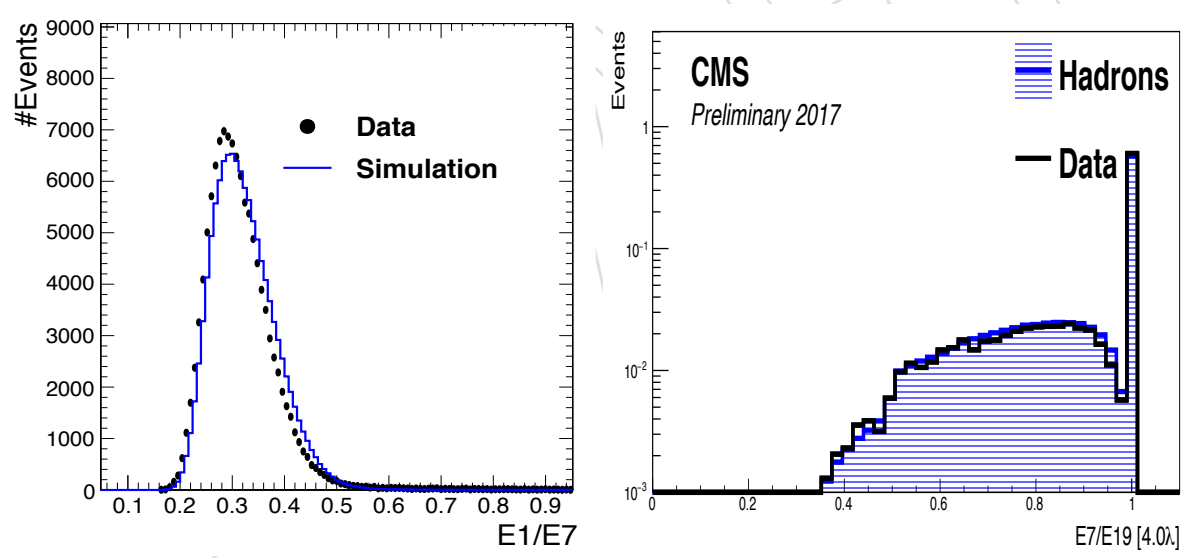

Fig. 7. E1/E7 on the left for $100 \mathrm{GeV}$ electrons at a depth of $10 X_{0}$ in the 2016 beam-tests and E7/E19 for $200 \mathrm{GeV}$ charged hadrons (20\% pions; $80 \%$ protons) measured at a depth of $4 \lambda$ in the 2017 beam-tests for data (points) and simulation (histogram).

\section{Conclusion}

An HGCAL prototype with $\mathrm{CE}-\mathrm{E}, \mathrm{CE}-\mathrm{H}(\mathrm{Si})$ and $\mathrm{CE}-\mathrm{H}(\mathrm{Scintillator})$ was constructed and operated for the first time in 2017. For the 2017 beam-tests, hadronic showers could be explored which was not possible in the 2016 beam-tests. SKIROC2CMS ASIC was used in the beam-tests done in 2017. MIP signals were observed in all the beam-tests performed during 2016 and 2017. Data and simulation comparison has been made for the shower-shape variables using electron and pion beams which shows reasonable agreement. Since July 2017, more beam-tests have been performed at CERN for which the analysis is still going on. For next year, the plan is to have a large number of $\mathrm{Si}$ modules tested $(>100)$.

\section{References}

1. D. Contardo, M. Klute, J. Mans, L. Silvestris and J. Butler, Technical Proposal for the Phase-II Upgrade of the CMS Detector, Tech. Rep. CERN-LHCC-2015-010. LHCCP-008. CMS-TDR-15-02 (Geneva, 2015), Upgrade Project Leader Deputies: Lucia Silvestris (INFN-Bari), Jeremy Mans (University of Minnesota) Additional contacts: Lucia.Silvestris@cern.ch, Jeremy.Mans@cern.ch.

2. L. Mastrolorenzo, submitted to Intl. Journal of Modern Physics: Conference Series.

3. S. Callier, F. Dulucq, C. de La Taille, G. Martin-Chassard and N. Seguin-Moreau, Journal of Instrumentation 6, p. C12040 (2011).

4. J. Borg, S. Callier, D. Coko, F. Dulucq, C. de La Taille, L. Raux, T. Sculac and D. Thienpont, Journal of Instrumentation 12, p. C02019 (2017).

5. S. Jain, Journal of Instrumentation 12, p. C03011 (2017).

6. C. Adloff et al., JINST 5, p. P05004 (2010). 